Preprint

UCRL-JC- 150832

\title{
Modification of the Coherence
}

Properties of a Laser Beam

Propagating through a Plasma

and its Consequences for

Stimulated Scattering Instabilities

C. Labaune, H.A. Baldis, H. Bandulet, S. Depierreux, J. Fuchs, P. Michel, D. Pesme

This article was submitted to European Conference on Laser Interaction with Matter (ECLIM2002), October 7-11, 2002, Moscow, Russia

U.S. Department of Energy

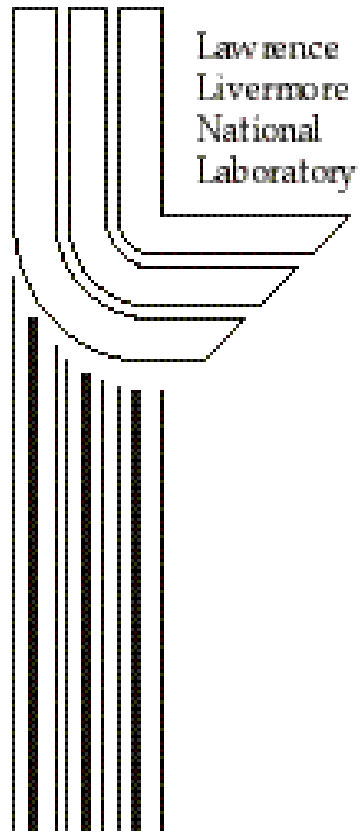

November 19, 2002 


\section{DISCLAIMER}

This document was prepared as an account of work sponsored by an agency of the United States Government. Neither the United States Government nor the University of California nor any of their employees, makes any warranty, express or implied, or assumes any legal liability or responsibility for the accuracy, completeness, or usefulness of any information, apparatus, product, or process disclosed, or represents that its use would not infringe privately owned rights. Reference herein to any specific commercial product, process, or service by trade name, trademark, manufacturer, or otherwise, does not necessarily constitute or imply its endorsement, recommendation, or favoring by the United States Government or the University of California. The views and opinions of authors expressed herein do not necessarily state or reflect those of the United States Government or the University of California, and shall not be used for advertising or product endorsement purposes.

This is a preprint of a paper intended for publication in a journal or proceedings. Since changes may be made before publication, this preprint is made available with the understanding that it will not be cited or reproduced without the permission of the author.

This report has been reproduced directly from the best available copy.

Available electronically at http://www.doc.gov/bridge

Available for a processing fee to U.S. Department of Energy

And its contractors in paper from

U.S. Department of Energy

Office of Scientific and Technical Information

P.O. Box 62

Oak Ridge, TN 37831-0062

Telephone: (865) 576-8401

Facsimile: (865) 576-5728

E-mail: reports@adonis.osti.gov

Available for the sale to the public from

U.S. Department of Commerce

National Technical Information Service

5285 Port Royal Road

Springfield, VA 22161

Telephone: (800) 553-6847

Facsimile: (703) 605-6900

E-mail: orders@ntis.fedworld.gov

Online ordering: http:/ /www.ntis.gov/ordering.htm

\section{OR}

Lawrence Livermore National Laboratory

Technical Information Department's Digital Library

http://www.llnl.gov/tid/Library.html 


\title{
Modification of the coherence properties of a laser beam propagating through a plasma
}

\section{and its consequences for stimulated scattering instabilities}

\author{
C. Labaune ${ }^{1}$, H.A. Baldis ${ }^{2}$, H. Bandulet ${ }^{1}$, S. Depierreux ${ }^{3}$, J. Fuchs ${ }^{1}$, P. Michel ${ }^{1}$, D. Pesme ${ }^{4}$

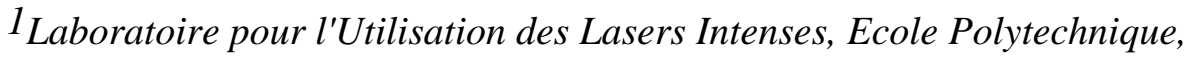 \\ Centre National de la Recherche Scientifique, 91128 Palaiseau cedex, France \\ 2 Institute for Laser Science and Applications (ILSA) \\ Lawrence Livermore National Laboratory, POB 808, Livermore CA 94550, USA \\ ${ }^{3}$ CEA-DIF, BP 12, 91680 Bruyères-Le-Châtel, France \\ ${ }^{4}$ Centre de Physique Théorique, Ecole Polytechnique, 91128 Palaiseau Cedex, France
}

Keywords : laser-plasma interaction, inertial confinement fusion, parametric instabilities, plasma induced incoherence

\begin{abstract}
The control of coherence is a critical issue for the high-power lasers used in inertial confinement fusion (ICF). The level of coherence is an important parameter for the control of the light intensity distribution as well as the growth rate of parametric instabilities. Over the past few years, experimental and theoretical studies have evidenced the ability of an underdense plasma to reduce the spatial and temporal coherence of an intense laser beam propagating through it. As any process affecting laser propagation, plasma-induced incoherence appears fundamental for ICF for it can impact on wave-coupling conditions.

We present results obtained with the six-beam LULI laser facility, in the nanosecond regime, showing direct evidences of the reduction of spatial and temporal coherence of an initially RPP-smoothed laser beam after propagation through a preformed plasma. Plasma induced incoherence (PII) proceeds from several mechanisms which include self-focusing and filament instabilities and non-linear coupling between self-focusing and forward stimulated Brillouin scattering (FSBS). Part of these experiments was dedicated to the understanding of the physical mechanisms involved in PII, as the break up of a single hot spot and the existence of ion acoustic waves having small wave vectors transverse to the interaction beam which are produced in the PII processes. The spatial and temporal characteristics of these waves give a unique access to the influence of PII on stimulated Brillouin and Raman scattering.
\end{abstract}

\section{INTRODUCTION}

The coupling and the propagation of the laser beams in the plasma surrounding the pellet must be very well controlled to succeed in producing energy in inertial confinement fusion experiments. This implies a good understanding of the laser-plasma interaction mechanisms to keep the non linear processes at a low level. The coherence of the laser beam is an important parameter for the growth of parametric instabilities. Optical smoothing techniques, which increase spatial and temporal incoherence of laser beams, have been proposed and implemented to reduce the growth of these non linear processes ${ }^{1}$. Some contradictory results in different experiments were not completely understood, because an important effect was not taken into account. This effect has been discovered 
recently and improves a lot our understanding of laser-plasma interaction physics. It concerns the capability of a plasma to reduce the temporal and spatial coherence of a laser beam propagating through it. This reduced coherence leads to an overall "plasma-induced smoothing" of the laser beam and modifies the non linear processes growths.

Theoretical and numerical studies of plasma induced incoherence (PII) have shown that this process can arise as the result of forward scattering processes such as non stationary filamentation, stimulated Brillouin scattering and the resonant instability of filaments ${ }^{2}$. PII generates a decrease of the effective f-number of the beam and a reduction of its coherence time. Experimental signatures of the increase of the angular divergence and of the spectral width have been observed ${ }^{3}$. We have carried out an experiment with the six-beam LULI laser facility to study the temporal and spatial reduction of the coherence of a laser propagating through a preformed plasma, as well as the physical mechanisms which were involved and the consequences of PII on stimulated Brillouin and Raman scattering (SBS and SRS). The interaction beam was either smoothed with a random phase plate or corrected from aberrations using an adaptative optics and so focused close to the limit of diffraction.

\section{EXPERIMENTAL SET-UP}

The experiments were performed with the 6-beam LULI facility at Ecole Polytechnique, using three $0.53 \mu \mathrm{m}$ beams to preform and heat the plasma from a thin exploded plastic foil, one or two $1.053 \mu \mathrm{m}$ beams as interaction beams, and one $0.35 \mu \mathrm{m}$ beam as a probe beam for Thomson scattering diagnostics. The set-up is shown in figure 1 . The main interaction beam was focused with an $\mathrm{f} / 6$ lens either through a random phase plate, producing a focal spot diameter of $320 \mu \mathrm{m}$ (containing $62 \%$ of the total energy), and maximum average intensity of $8 \times 10^{13} \mathrm{~W} / \mathrm{cm}^{2}$, or after correction of its phase front using a deformable mirror. With the adaptative optics, the focal spot diameter was $10 \mu \mathrm{m}$, full width at half maximum (FWHM), with a maximum peak intensity of $2 \times 10^{16} \mathrm{~W} / \mathrm{cm}^{2}$.

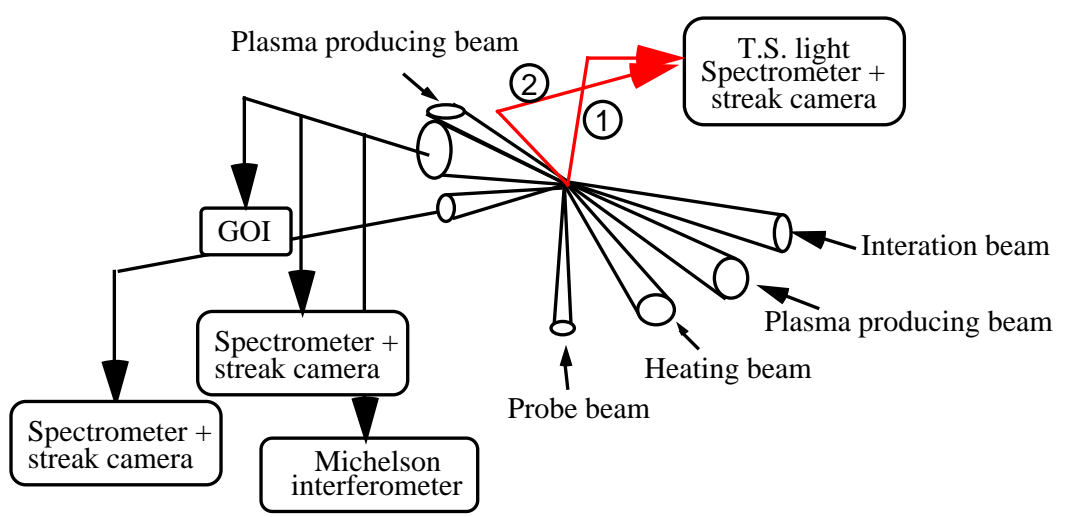

Figure 1 : Experimental beam configuration and diagnostics

The main diagnostics were based on the analysis of the forward scattered light at different angles and timeresolved spectra of Thomson scattered light off ion acoustic waves. The forward scattered light was analysed through 2D time-integrated far field images, time-resolved near field images, a Michelson interferometer and through a spectrometer coupled with a streak camera providing time-resolved spectra. The light was collected with an $f / 3$ lens, with a larger aperture than the focusing lens. The Thomson scattered light with frequency close to the one of the probe beam, $3 \mathrm{w}$, was collected in two directions, at $9^{\circ}\left( \pm 2^{\circ}\right)$ or at $20^{\circ}\left( \pm 5^{\circ}\right)$ from the probe beam axis. The light collected at $9^{\circ}$ has been scattered off ion acoustic waves having small wave vector of $0.6 \mathrm{k}_{0}\left(\mathrm{k}_{0}\right.$ being the wave vector of the interaction beam) and propagating transversely to the interaction beam axis. The light collected at $20^{\circ}$ has been scattered off ion acoustic waves associated with backward SBS of the interaction beam. 
The plasma was preformed by irradiating a thin micro disk of plastic with two $0.53 \mu \mathrm{m}$ beams from each side, $1.6 \mathrm{~ns}$ before the arrival of the interaction beam. The initial foil thickness and the laser energies were chosen so the foil exploded a short time before the arrival of the interaction beam, producing a quasi parabolic density profile. The preformed plasma has been characterised with various techniques. The electron temperatures ranged between 0.5 and $0.7 \mathrm{keV}$ during the interaction pulse. The electron density at the top of the plasma profile decreased exponentially in time from $0.3 \mathrm{n}_{\mathrm{c}}$ to $0.07 \mathrm{n}_{\mathrm{c}}$ ( $\mathrm{n}_{\mathrm{c}}$ is the critical density for $1.053 \mu \mathrm{m}$ light). The typical scale length of the inverse parabolic profile of the plasma was $700 \mu \mathrm{m}$.

\section{INDUCED SPATIAL INCOHERENCE}

A first evidence of the decrease of the spatial coherence had been obtained from the diffraction patterns of an object in the beam after propagation through the preformed plasma. The diffraction fringes produced by the edges of the element of the RPP placed just before the chamber on the interaction beam looked very clear after propagation through vacuum and became blurred when propagating through the plasma at high laser intensities. This blurring was interpreted by the superposition of slightly shifted patterns produced by enhanced angular spreading of the laser light ${ }^{4}$.

Another signature of the induced spatial incoherence was obtained from the temporal evolution of the near field pattern of the transmitted light, recorded with a gated optical imager (GOI), as shown in figure 2. The time of integration was $80 \mathrm{ps}$. One can clearly see an increase of the angular distribution after the peak of the pulse, compared to the one recorded early in the pulse. The angular distribution was much narrower when the incident intensity was reduced.

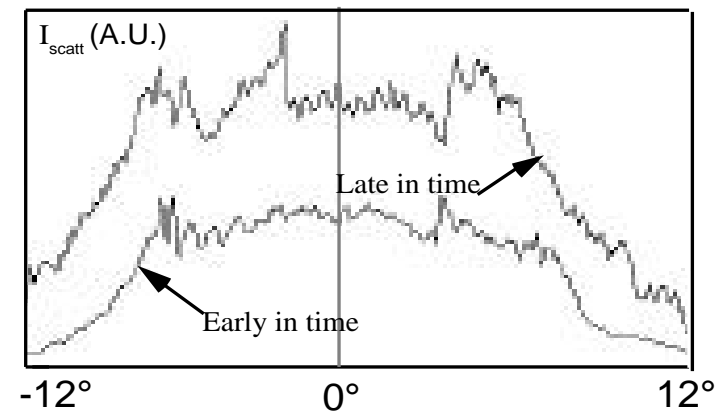

Figure 2: Angular diagram of the transmitted light, early and late during the laser pulse

\section{INDUCED TEMPORAL INCOHERENCE}

Evidence of induced temporal incoherence was obtained from the spectra of the scattered light which exhibit, at high intensity, both large broadening and large red-shifts. A typical time-resolved spectrum at $0-10^{\circ}$ is shown in figure 3 . The spectrum exhibits two spectral components : a thin component with a shift in agreement with the value given by classical forward SBS, and a second component which is red-shifted by a few Angstroms, broad, with temporal and spectral structures, starts later than the first component, and lasts until the end of the laser pulse.

Its shift decreases as a function of time. The shift, width, duration and amplitude of the red-shifted wing all increase with incident intensity. The traces of the two spectral components are shown as a function of time in figure 4 . The 
first component starts early in time and stops before the end of the pulse and exhibits a smooth temporal behaviour. The second component starts later and exhibits lots of temporal structures.

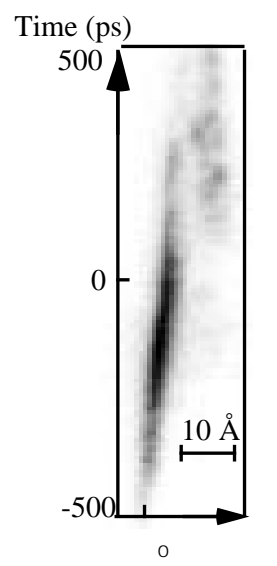

Figure 3 : Time-resolved spectrum of the transmitted light collected at $3^{\circ}-10^{\circ}$ from the laser axis

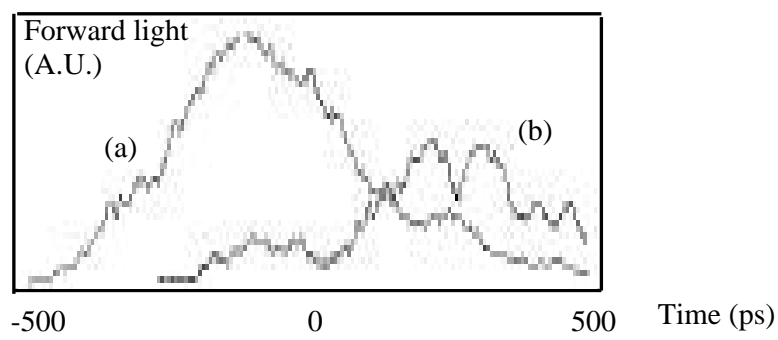

Figure 4 : Traces of the two spectral components of the forward scattered spectrum :

(a) small shift component ; (b) red-shifted component

The temporal coherence can be deduced from the time-resolved spectra using the Wiener-Khintchine theorem which states that the Fourier transform of the power spectrum is equal to the variation of the visibility as a function of time. This is reported in reference 5. To complete this work, direct measurements of the coherence time were obtained with a Michelson interferometer. Time-resolved interference patterns of the forward scattered light were recorded as a function of the interaction laser intensity and the delay between the two arms of the interferometer. We observed in figure 5 that, at an intensity of $10^{14} \mathrm{~W} / \mathrm{cm}^{2}$ and a delay of $8 \mathrm{ps}$, the fringes, which can be clearly seen at the beginning of the pulse, get blurred around the peak of the pulse and recover a good visibility at the end of the pulse. At an intensity of $10^{13} \mathrm{~W} / \mathrm{cm}^{2}$ and with the same delay, the blurring appears later in time and lasts for a shorter time. At lower intensity, $10^{11} \mathrm{~W} / \mathrm{cm}^{2}$ the fringes stay clear for all the pulse duration.

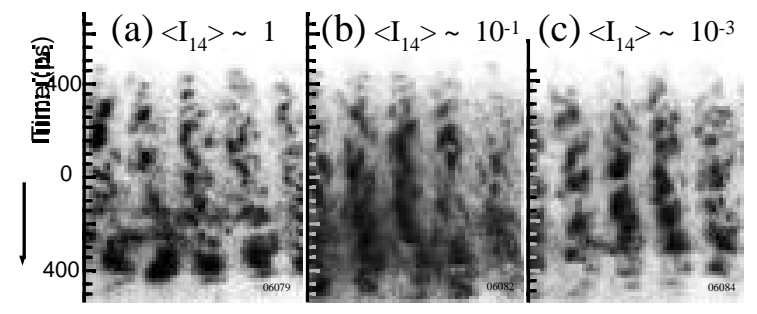

Fig.5 : Time-resolved fringe patterns in the output of the Michelson interferometer for light scattered at $7^{\circ}$ from the incident axis (outside the incident cone) after propagation through the preformed plasma for three laser intensities : $\left\langle\mathrm{I}_{14}>\sim 1\right.$ in (a), $10^{-1}$ in (b) and $10^{-3}$ in (c).

Using both diagnostics, we determined the coherence time of the output interaction beam for a wide range of laser and plasma conditions. The coherence time decreases as the laser intensity or the plasma density increase. The minimum coherence time that we observe was $\sim 4$ ps. 


\section{PHYSICAL MECHANISMS INVOLVED IN PLASMA INDUCED INCOHERENCE}

Theoretical work has shown that PII may be the result of coupled forward scattering processes, including self-focusing, filamentation, stimulated Brillouin scattering (SBS) and the resonant instability of filaments. Many features of the experimental results are in agreement with these processes. The red-shifted component observed in the forward scattered light can be explained by forward SBS growing in high intensity self-focused speckles, where the rapidly decrease of density could be responsible of the large red shift by self phase modulation. The instability of filaments can lead to the filament destruction and consequently detrapping of the light from the channel, resulting in a spiky behaviour of this light. This is what is observed for the red-component in figure 4 . In experiments with crossed beam irradiation ${ }^{6}$ the plasma induced incoherence was stronger and the bursts were also more contrasted (see Fig. 8).

An experiment was specially designed to study the evolution of the intensity distribution of a single filament propagating through the plasma. We used a deformable mirror and an adaptative optic loop to focus the interaction beam close to the diffraction limit. We varied the single hot spot intensity to cover the range of speckle intensities of the RPP beam. Time-integrated far field images were recorded to study the intensity distribution in the vacuum or after propagation of the beam through the preformed plasma. Beam break up was observed for intensities larger than $10^{13} \mathrm{~W} / \mathrm{cm}^{2}$ as can be seen in figure 6 . This means that many of the high intensity speckles of the RPP beam go through self-focusing and break up which are responsible for the observed PII.

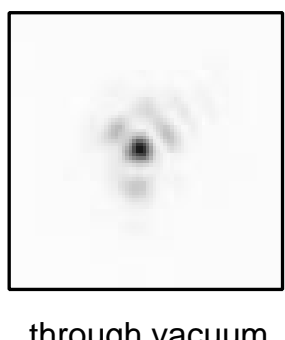

through vacuum
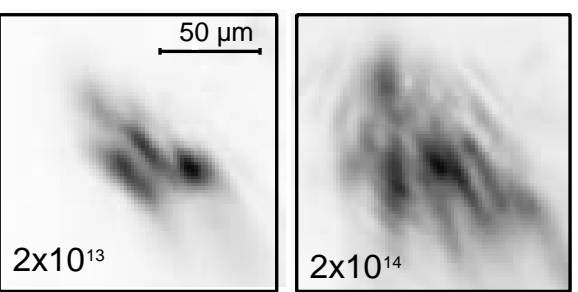

through a preformed plasma

Figure 6 : Far field intensity distribution through vacuum and through a preformed plasma for two laser intensities

Another experiment was designed to observed the ion acoustic waves which develop in the self focused speckles. From the numerical simulations, these waves have small wave vectors transverse to the interaction beam. Using a Thomson scattering diagnostic, we have observed these waves as well are their spatial and temporal behaviour. This is exposed in another paper of these proceeding ${ }^{7}$. Good correlation with the other signatures of PII gave us a good confidence on the participation of these ion acoustic waves in PII.

\section{CONSEQUENCES OF PII ON STIMULATED SCATTERING INSTABILITIES}

The plasma induced incoherence is predicted to have a strong effect on parametric instabilities ${ }^{2,8}$. The Thomson diagnostic demonstrated that ion acoustic waves associated with PII were located in a different part of the density profile along the laser propagation axis compared to the location of ion acoustic waves associated with backward SBS. This is shown in figure 7. They also show that the ion acoustic waves associated with backward SBS disappeared in time when the ion acoustic waves associated with plasma smoothing started to grow. This may be the first experimental evidence of the hindrance in space and time to the backward SBS due to plasma smoothing. 


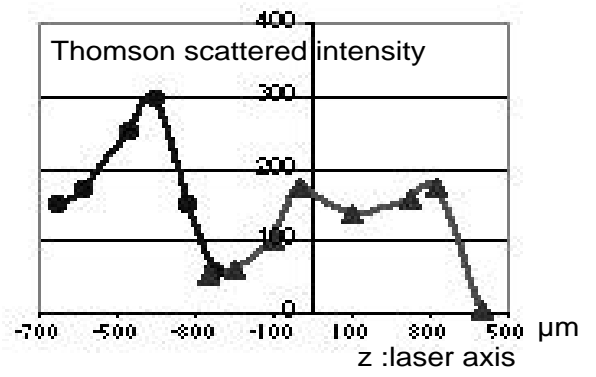

Figure 7 : Location of the ion acoustic waves associated with backward SBS (circles) and with plasma induced incoherence (triangles)along the laser axis.

In a second experiment, enhancement of extreme forward scattered light of a laser beam in the presence of a second, identical, laser beam, crossing the first one at $22,5^{\circ}$ has been observed ${ }^{6}$. The spectral analysis of the forward scattered light showed that the additional forward energy was added in a non linear way to the red-shifted component (Fig. 8). An overall increase of the forward scattered light of 2.3 has been measured. The large angle forward SBS between directions of two beams was triggered by single beam plasma induced smoothing effects. In addition to enhancing spectral width and angular spreading the forward SBS can contribute to the energy exchange between the beams. An effective plasma smoothing for the crossed beam irradiation was also responsible for the reduction of backward SBS reflectivity.

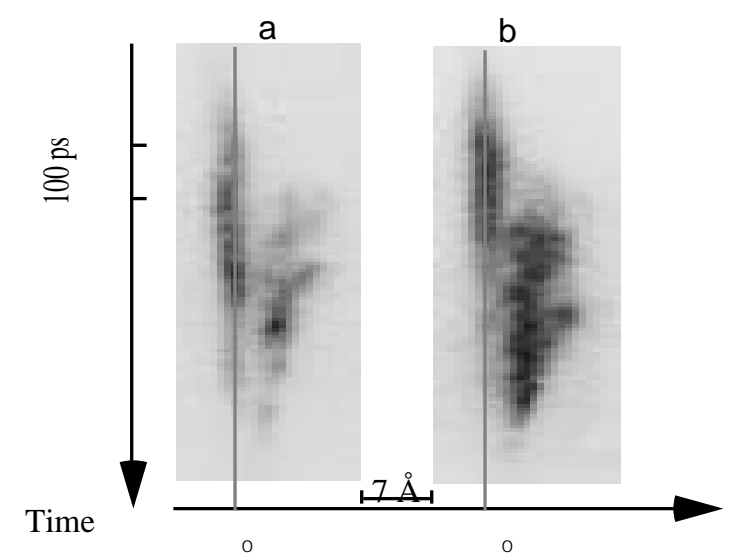

Figure 8 : Time-resolved spectra of forward scattered light in case of single beam irradiation (a) and in case of cross beam irradiation (b).

\section{CONCLUSION}

Complementary diagnostics have shown the reduction of spatial and temporal coherence of a laser beam after its propagation through a preformed plasma.. The results are in agreement with the principal trends of the physical mechanisms involved in PII, in particular concerning the effect of the laser intensity and the plasma electron density. Plasma induced incoherence may be important in the context of laser fusion by modifying the growth of parametric instabilities. 


\section{ACKNOWLEDGMENTS}

The authors gratefully acknowledge the support of A. Michard and the technical groups of LULI during the experiments. Part of this work was performed under the auspices of the U.S. Department of Energy by University of California Lawrence Livermore National Laboratory, through the Institute for Laser Science and Applications, under Contract No.W-7405-Eng-48.

\section{REFERENCES}

${ }^{1}$ Y. Kato, K. Mima, N. Miyanaga, S. Arinaga, Y. Kitagawa, M. Nakatsuka, C. Yamanaka, Phys. Rev. Lett. 53, 1057 (1984); R.H. Lehmberg, A.J. Schmitt and S.E. Bodner, J. Appl. Phys. 62, 2680 (1987).

${ }^{2}$ V.V. Eliseev, I. Ourdev, W. Rozmus, V.T. Tikhonchuk, C.E. Capjack, P.E. Young, Phys. Plasmas 4, 4333 (1997); A. Schmitt and B. Afeyan, Phys. Plasmas 5, 503 (1998) ; D. Pesme et al., Phys. Rev. Lett. 84, 278 (2000) ; G. Riazuelo and G. Bonnaud, Phys. Plasmas 7, 3841 (2000) ; A. Maximov et al., Phys. Plasmas 8, 1319 (2001) ; J. Myatt et al., Phys. Rev. Lett. 87, 255003 (2001).

${ }^{3}$ C. Labaune et al., C. R. Acad. Sci. Paris, t.1, Série IV, 727 (2000) ; P. Young et al., Phys. Plasmas 2, 2825 (1995) ; J. Moody et al., Phys. Rev. Lett. 83, 1783 (1999) ; J. Fuchs et al., Phys. Rev. Lett. 86, 432 (2001).

${ }^{4}$ C. Labaune et al. IFSA 1999, edited by C. Labaune, W.J. Hogan and K.A. Tanaka, 289 (1999).

${ }^{5}$ J. Fuchs, C. Labaune, H. Bandulet, P. Michel, S. Depierreux, H.A. Baldis, Phys. Rev. Lett. 88, 195003 (2002).

${ }^{6}$ C. Labaune et al. Phys. Rev. Lett. 85, 1658 (2000).

${ }^{7} \mathrm{H}$. Bandulet et al., same proceedings.

Ph. Mounaix et al., Phys. Rev. Lett. 85, 4526 (2000).

${ }^{8}$ C. Labaune et al. Phys. of Plasmas 6, 2048 (1999). 